\title{
INVARIANT CORNER DETECTION USING STEERABLE FILTERS AND HARRIS ALgORITHM
}

\author{
Mahesh $^{1}$ and Dr.M.V.Subramanyam ${ }^{2}$ \\ ${ }^{1}$ Research scholar, Department of ECE, MITS, Madanapalle, AP, India \\ vka4mahesh@gmail.com \\ ${ }^{2}$ Professor, Department of ECE, SREC, Nandyal, AP, India \\ mvsrajeyahoo.com
}

\begin{abstract}
This paper proposes a new invariant corner detection algorithm using steerable filters and Harris corner detection. The steerable filters have better orientation selectivity and multi-orientation image decomposition that provide a useful front-end for image-processing and computer vision applications. Corners, in image analysis, are important features for image registration, stereo matching, motion tracking and object recognition. These corners are referred as interest points or key points for image registration. In this paper, we compare the performance of SUSAN, Harris, and propose corner detectors in terms of consistency.
\end{abstract}

\section{KEYWORDS}

Corner detection, Steerable filters, SUSAN, Harris, Image registration, Consistency

\section{INTRODUCTION}

Corner points are formed from junctions of two or more edges. And usually an edge defines the boundary between two different objects or parts of the same object. The number of approaches exists for detecting corners. A successful corner detector should detect all the true corners. Further, with minimum number of false corners, the detected corners are to be robust to noise and invariant to resolution, scale and orientation.

Corner detector in Kitchen and Rosenfeld[3] method is based on the change of gradient direction along an edge contour multiplied by the local gradient magnitude. This method is suffers from missing junctions, poor localization and sensitive to noise.

Harris[1] corner detector method is based on assumption that the corners are associated with local maximum functions. Corner value of each pixel in the image computed using Harris detector. The corner value of a pixel is below a certain threshold is declared as a corner. This method gives good repeatability with rotation and change in illumination. It is suffers from loss in localization accuracy and sensitive to quantization noise. SUSAN[2] (Smallest Univalue Segment Assimilating Nucleus) method proposed by Smith and Brady. This detector is based on circular mask for corner and edge detection. SUSAN corner detector makes a better noise robustness and localization compared to previous algorithms, but has an average repeatability rate. We propose a corner detection using steerable filters and Harris in this paper. The detected corners in the image are invariant to rotation, scaling, and partially invariant to illumination change and 3D view point. Experimental results demonstrate that proposed algorithm is robust to rotation and scaling invariant.

DOI : 10.5121/sipij.2012.3509 
Signal \& Image Processing : An International Journal (SIPIJ) Vol.3, No.5, October 2012

\section{STEERABLE FILTERES}

Steerable filters, introduced by Freeman and Adelson [4], are spatial oriented filters that can be expressed using linear combinations of a fixed set of basis filters. If the transformation is a translation, then the filter is said to be shiftable or steerable in position; if the transformation is a rotation, then the filter is said to be steerable in orientation or commonly steerable and the basis filters are normally called steerable basis filters. Given a set of steerable basis filters, we can apply them to an image and since convolution is linear, we can interpolate exactly, from the responses of the basis filters, the output of a filter tuned to any orientation we desire.

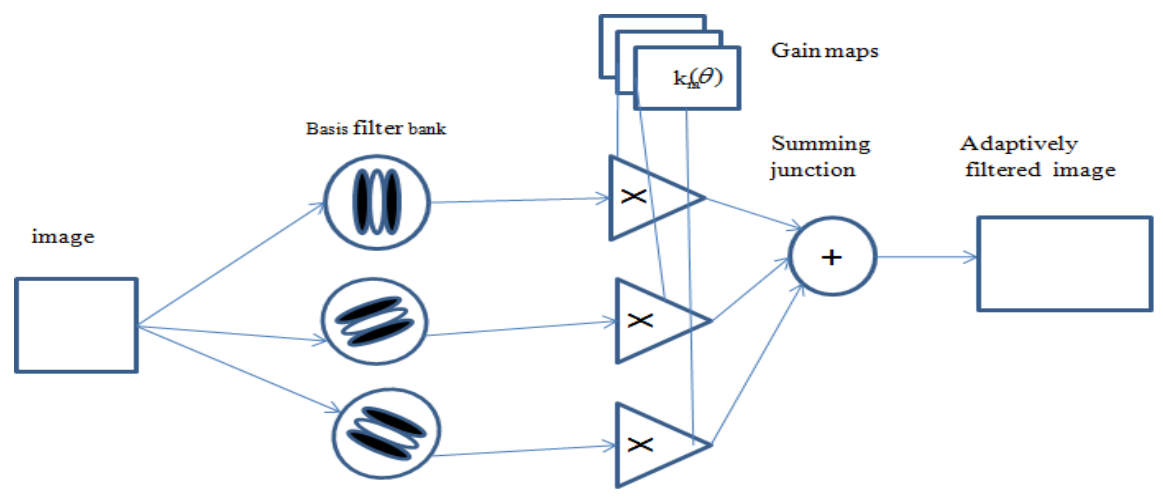

Figure1. Steerable filter block diagram

The basic idea is to generate a rotated filter from a linear combination of a fixed set of basis filters. Figure 1 shows a general architecture for steerable filters, which consists of a bank of permanent, dedicated basis filters that always convolve the image as it comes in. The outputs are multiplied by a set of gain masks, which apply the appropriate interpolation functions at each position and time. The final summation produces the adaptively filtered image.

The steerability condition is not restricted to derivative filters and could be expressed for any signal $f$ as:

$$
f^{\theta}(x, y)=\sum_{m=1}^{M} k_{m}(\theta) f^{\theta_{m}}(x, y)
$$

where $f^{\theta}(x, y)$ is the rotated version of $f$ by an arbitrary angle $\theta, k_{m}(\theta)$ are the interpolation functions, $f^{\theta_{m}}(x, y)$ are the basis functions and $M$ the number of basis functions required to steer the function $f(x, y)$.

To determine the conditions under which a given function satisfies the steering condition in Eq. (1), let us work in polar coordinates $\left(r=\sqrt{x^{2}+y^{2}}\right.$ and $\left.\phi=\arg (x, y)\right)$.

The function $f$ could be expressed as Fourier series in polar angle $\phi$ :

$$
f(r, \phi)=\sum_{n=-N}^{N} a_{n}(r) e^{j n \phi}
$$

where $\mathrm{j}=\sqrt{-1}$ and $N$ is the discrete length of coefficients. It has been demonstrated in [5] that the steering condition in Eq. (1) is satisfied for functions expandable in the form of Eq. (2) if and if only the interpolation function $k_{m}(\theta)$ are solution of:

$c_{n}(\theta)=\sum_{m=-1}^{M} k_{m}(\theta)\left(c_{n}(\theta)\right)^{m}$ 
where $c_{n=} e^{j n \theta}$, and $n=\{0, \ldots, N\}$.

From of Eq.(3), $f^{\theta}(r, \phi)$ is expressed as:

$f^{\theta}(r, \phi)=\sum_{m=1}^{M} k_{m}(\theta) g_{m}(r, \phi)$

where $g_{m}(r, \phi)$ can be any set of functions.

It has been also demonstrated that the minimum number $M$ of basis functions required to steer

$f(r, \phi)$ is equal to the number of non-zero Fourier coefficients $a_{n}(r)$.

\section{HARRIS CORNER DETECTOR}

The Harris corner detector method is based on an underlying assumption that corners are associated with the maxima of the local function. The computations are based on first derivatives so; it is less sensitive to noise. For each pixel in the image $I(x, y)$, the detector computes a corner value $\mathrm{C}$. When the value of $\mathrm{C}$ is below a certain threshold, the pixel is declared as a corner. The value of $\mathrm{C}$ is computed from the intensity gradients in the $x$ and $y$ directions as follows:

$$
\begin{gathered}
\mathrm{M}=\left(\begin{array}{cc}
I_{x}{ }^{2} & I_{x} I_{y} \\
I_{x} I_{y} & I_{y}{ }^{2}
\end{array}\right) \\
\mathrm{C}=\operatorname{det}(M)-k \operatorname{Tr}(M)
\end{gathered}
$$

where $I_{x}$ and $I_{y}$ denote the first derivative of the point $I(\mathrm{x}, \mathrm{y})$ in the image along the $x$ and $y$ directions respectively. The derivatives are determined by convolving the image by a kernel of the correspondent derivative of a Gaussian, and $k$ is a constant with a generally assumed value of 0.04. This detector is invariant to noise, changes in illumination and rotation.

\section{SUSAN CORNER DETECTOR}

This detector does not use spatial derivatives nor smoothes the image. Instead, a circular mask is applied around every pixel, and the greyscale values of all the pixels within the mask are compared to that of the centre pixel (the "nucleus"). Calculate the number of pixels within the circular mask which have similar brightness to the nucleus. This circular mask applied to different positions of a black rectangle with the USAN shown red colour in figure 2.

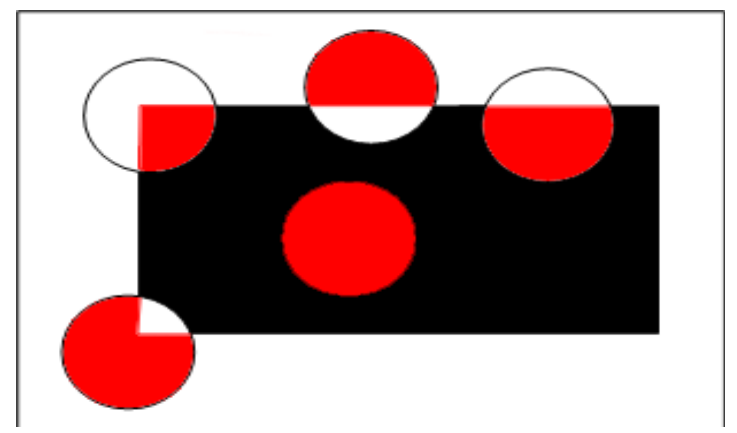

Figure 2. USAN for different circular masks on a uniform rectangle 
Signal \& Image Processing : An International Journal (SIPIJ) Vol.3, No.5, October 2012

It is observed that the USAN becomes smaller as it approaches an edge and this reduction is stronger at corners and SUSAN can thus be used for both line and edge detection. This corner detector computes fast, with good repeatability rate. This simple detector that is invariant to rotation, changes in illumination but and however it is sensitive noise.

\section{Proposed Method}

The Kitchen and Rosenfeld, Harris, and SUSAN corner detection methods detect noise as false corners and miss some fine features or true corners. We propose new corner detection using the steerable filters and Harris algorithm as follows:

1. Decomposition of an image with different orientations using steerable filters

2. Detect the corners in each orientation using Harris algorithm.

3. Combining all detected corners by performing logical or operation.

4. Applying dilation to the combining nearby corners to make them into one.

5. Find the centroid of step 4 and identified as corner.

We propose a corner detection algorithm as shown in figure 3 that uses the steerable filters. The experiments carried out with matlab make it clear that if the number of orientations is four it gives better localization and minimum number of false corners detection.

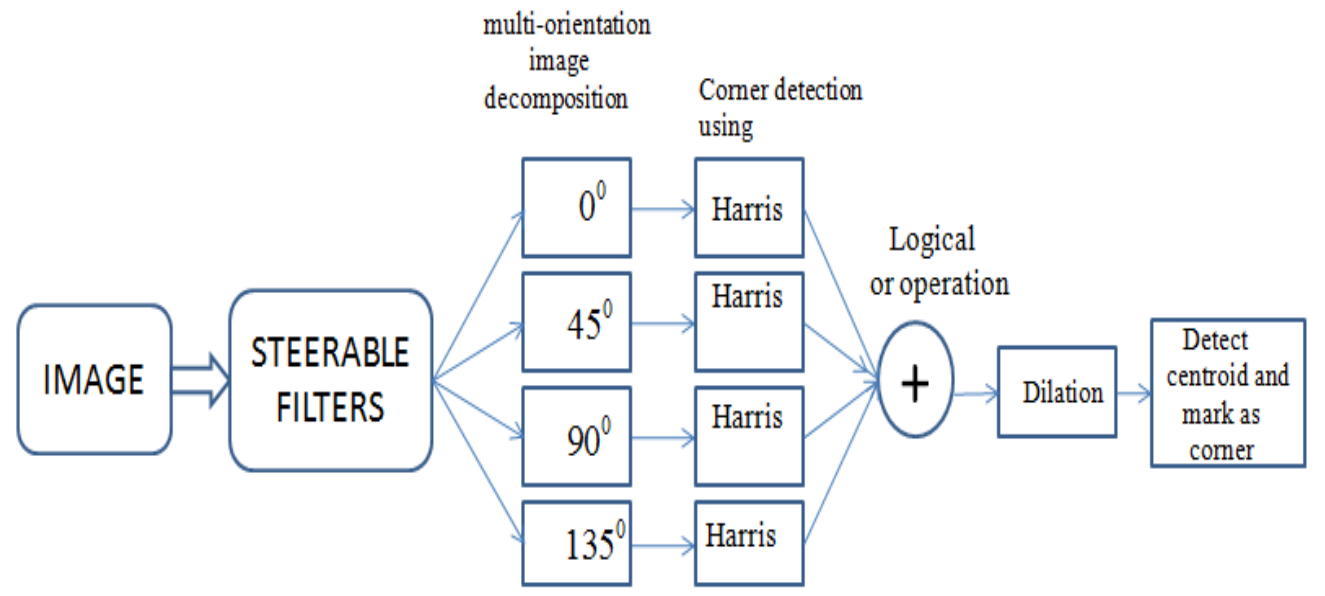

Figure 3. Block diagram proposed algorithm

\section{RESULT AND DISCUSSION}

The proposed corner detection algorithm is tested for different images in this section. Figures 4(a) to 4(i) shows the detected corner results. 
Signal \& Image Processing : An International Journal (SIPIJ) Vol.3, No.5, October 2012

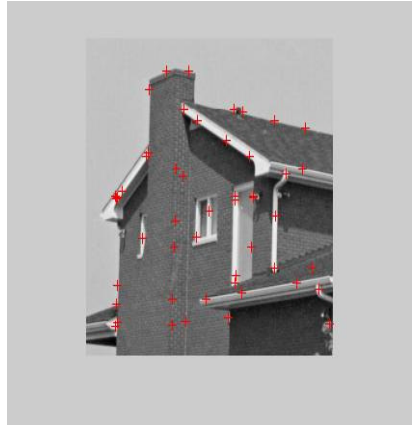

(a) susan

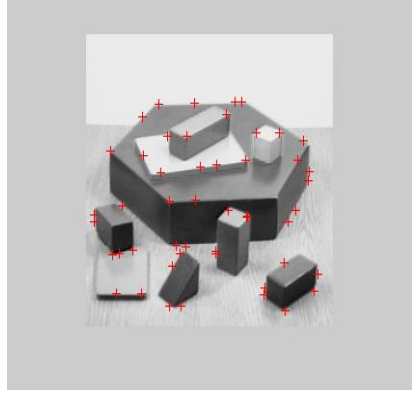

(d) susan

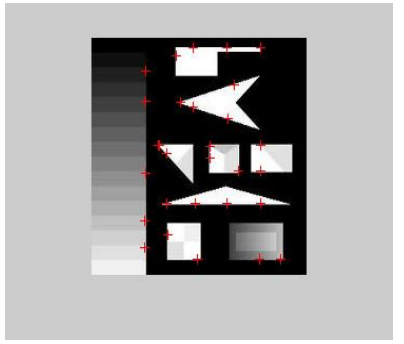

(g) susan

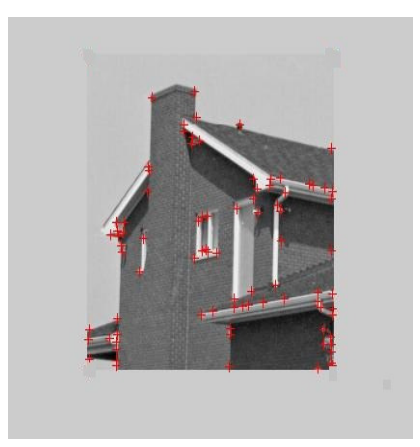

(b) harris

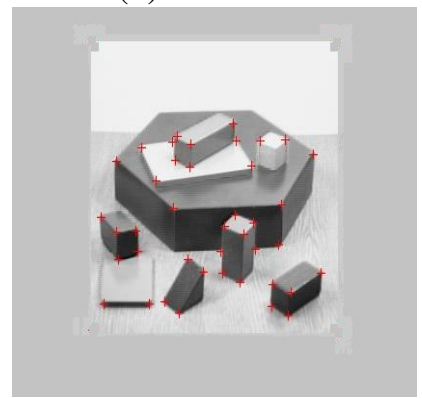

(e) harris

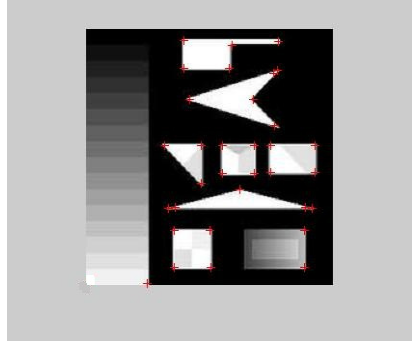

(h) harris

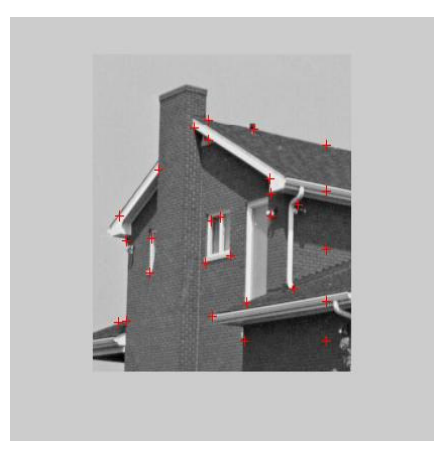

(c) proposed

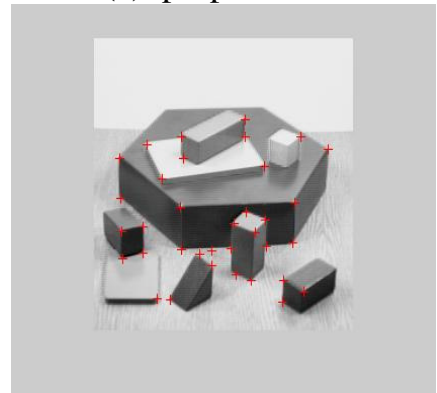

(f) proposed

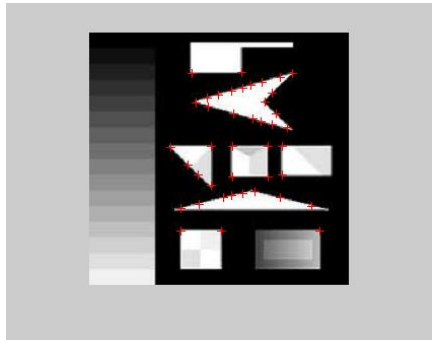

(i) proposed

Figures 4(a)-4(i) : corner detection on test images "house", "block" and "sample"

Table1. Corner results for the "House" image

\begin{tabular}{|c|c|c|c|c|c|}
\hline Rotation & Detector & $\begin{array}{c}\text { Detected } \\
\text { corners }\end{array}$ & $\begin{array}{c}\text { True } \\
\text { corners }\end{array}$ & $\begin{array}{c}\text { Missed } \\
\text { corners }\end{array}$ & $\begin{array}{c}\text { False } \\
\text { corners }\end{array}$ \\
\hline \multirow{3}{*}{$0^{\circ}$} & Susan & 49 & 20 & 17 & 22 \\
\cline { 2 - 6 } & Harris & 89 & $\mathbf{3 5}$ & $\mathbf{6}$ & $\mathbf{3 2}$ \\
\cline { 2 - 6 } & Proposed & 37 & $\mathbf{2 5}$ & $\mathbf{9}$ & $\mathbf{1 2}$ \\
\hline \multirow{3}{*}{$30^{\circ}$} & Susan & 82 & 12 & 21 & 32 \\
\cline { 2 - 6 } & Harris & 207 & $\mathbf{3 7}$ & $\mathbf{8}$ & $\mathbf{1 6}$ \\
\cline { 2 - 6 } & Proposed & 65 & $\mathbf{3 5}$ & $\mathbf{1 4}$ & $\mathbf{3}$ \\
\hline \multirow{3}{*}{$60^{\circ}$} & Susan & 80 & 13 & 20 & 32 \\
\cline { 2 - 6 } & Harris & 221 & $\mathbf{3 6}$ & $\mathbf{7}$ & $\mathbf{2 2}$ \\
\cline { 2 - 6 } & Proposed & 73 & $\mathbf{3 8}$ & $\mathbf{1 2}$ & $\mathbf{6}$ \\
\hline
\end{tabular}


Signal \& Image Processing : An International Journal (SIPIJ) Vol.3, No.5, October 2012

Table2. Corner results for the "Block" image

\begin{tabular}{|c|c|c|c|c|c|}
\hline \multirow{2}{*}{ Rotation } & Detector & $\begin{array}{c}\text { Detected } \\
\text { corners }\end{array}$ & $\begin{array}{c}\text { True } \\
\text { corners }\end{array}$ & $\begin{array}{c}\text { Missed } \\
\text { corners }\end{array}$ & $\begin{array}{c}\text { False } \\
\text { corners }\end{array}$ \\
\hline \multirow{3}{*}{$0^{\circ}$} & Susan & 49 & 30 & 28 & 19 \\
\cline { 2 - 6 } & Harris & 45 & $\mathbf{4 0}$ & $\mathbf{1 8}$ & $\mathbf{1}$ \\
\cline { 2 - 6 } & Proposed & 43 & $\mathbf{3 9}$ & $\mathbf{1 8}$ & $\mathbf{4}$ \\
\hline \multirow{3}{*}{$30^{\circ}$} & Susan & 78 & 17 & 37 & 21 \\
\cline { 2 - 6 } & Harris & 224 & $\mathbf{2 1}$ & $\mathbf{3 4}$ & $\mathbf{1}$ \\
\cline { 2 - 6 } & Proposed & 68 & $\mathbf{3 2}$ & $\mathbf{2 6}$ & $\mathbf{1}$ \\
\hline \multirow{3}{*}{$60^{\circ}$} & Susan & 79 & 19 & 38 & 20 \\
\cline { 2 - 6 } & Harris & 222 & $\mathbf{2 4}$ & $\mathbf{3 4}$ & $\mathbf{1}$ \\
\cline { 2 - 6 } & Proposed & 71 & $\mathbf{3 5}$ & $\mathbf{2 3}$ & $\mathbf{3}$ \\
\hline
\end{tabular}

\subsection{Performance Analysis of Corner Detection}

The performance is evaluated in terms of number of true, false, and missed corners detected by the algorithms. Tables 1 and 2 show the detection of corner results summary for rotation $0^{\circ}, 30^{\circ}$ and $60^{\circ}$. The proposed method result is compared with SUSAN and Harris. Initially, an experiment is conducted to detect corners for orginal "house" "block" and " sample" images. Next, orginal image is rotated with an angle from $+90^{\circ}$ to $-90^{\circ}$. It has been observed that proposed method detected minimum number of false and missed corners and, stable and well loclalizd true corners even after rotation of images.

The stability of corner detection alogorithms is measure using consistency of corner numbers (CCN)[8]. Consistency means the detected corner numbers should be invariant to the combination of noise, rotation, uniform or nonuniform scaling and affine transform. Definition of consistency of corner numbers is as follows:

$$
\mathrm{CCN}=100 \times 1.1^{-|N t-N o|}
$$

No - Detected corner numbers in orginal image.

$N t$ - Corner numbers in the transformed image.

$\mathrm{CCN}$ should be near to $100 \%$ for stable corner detectors. CCN is near to zero for corner detectors with too many false corners. It is noticed that $\mathrm{CCN}$ measures only number of detected corners without regard to whether they are correct or not.

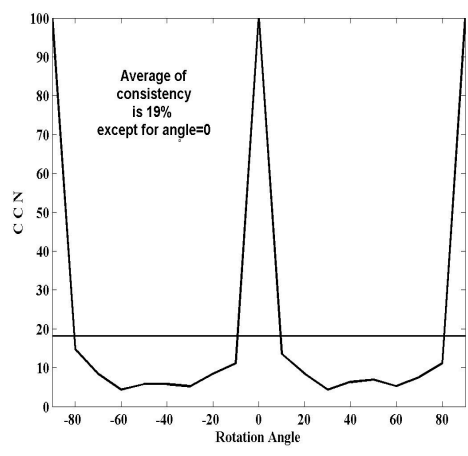

(a) susan

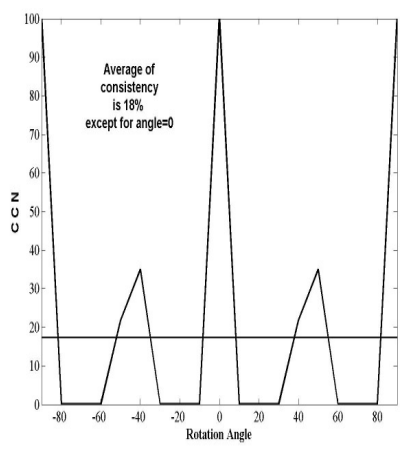

(b) harris

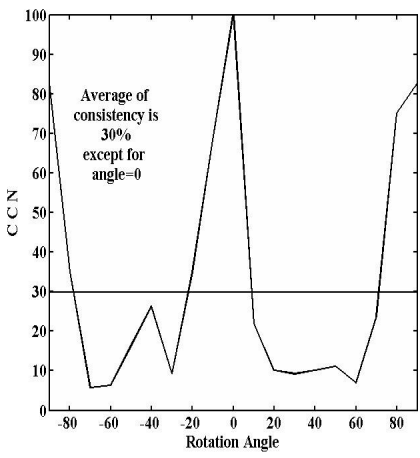

(c) proposed

Figures 5(a)-5(c): consistency of corner numbers for rotation "house" image 
Signal \& Image Processing : An International Journal (SIPIJ) Vol.3, No.5, October 2012

Figure 5 shows experimental results of $\mathrm{CCN}$ values for house image. First, corners are detected for original image. Next, original image is rotated with an angle in steps of $10^{\circ}$ variations between $-90^{\circ}$ and $+90^{\circ}$. The performances of the proposed algorithm makes better than SUSAN and Harris.

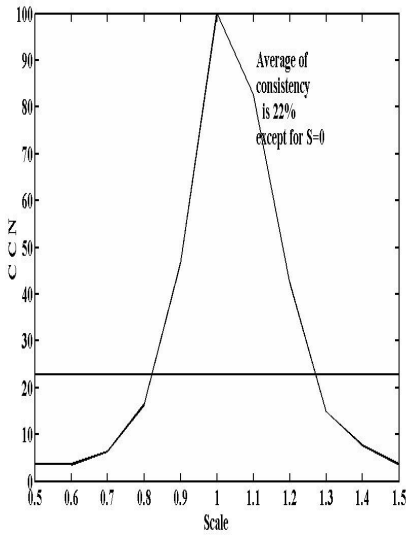

(a) susan

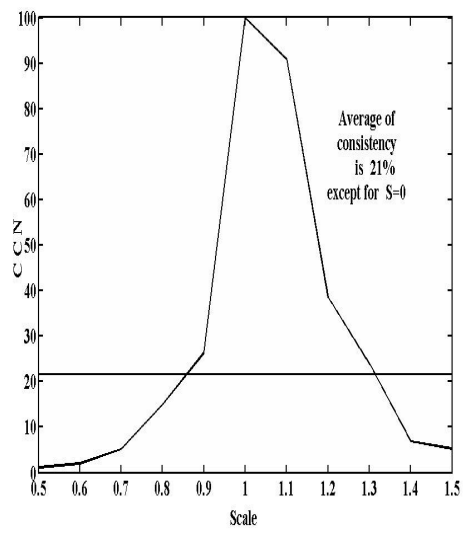

(b) harris

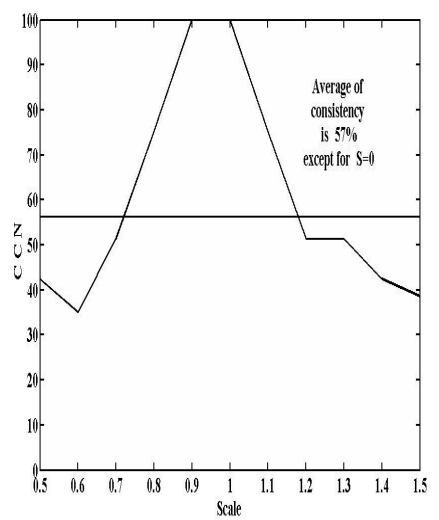

(c) proposed

Figures 6(a)-6(c): consistency of corner numbers for uniform scaling "house" image

Figure 6 shows experimental results of $\mathrm{CCN}$ values for house image. First corners are detected for original image. Next, original image is uniformly scaled with variations between 0.5 and 1.5. It shows that the proposed algorithm performances better than SUSAN and Harris.

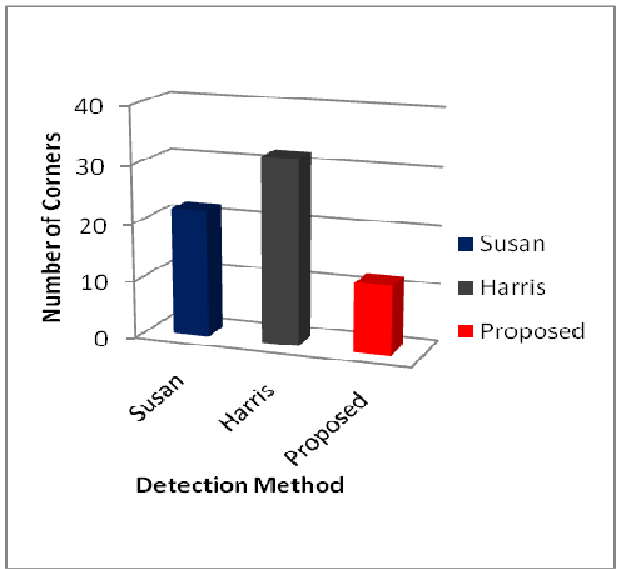

(a): Number of False corners detected

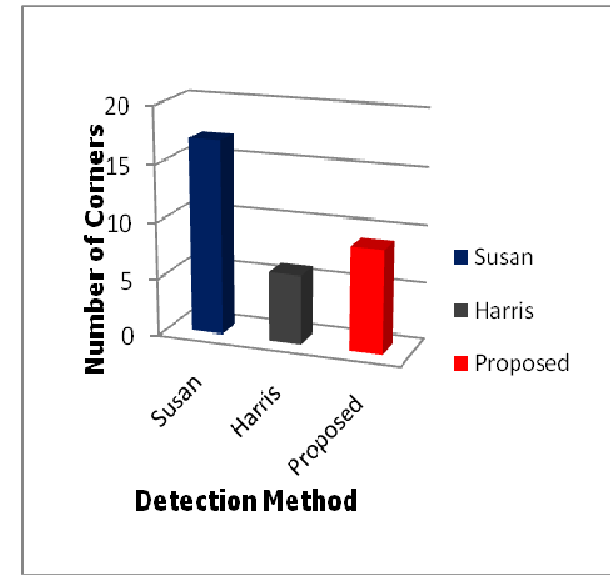

(b): Number of Missed corners detected

Figures 7(a)-(b): compare "house" image corners results

Figure 7 shows corner results comparision for "house" image. It is observed that the proposed method detects the minimum number of false and missed corners.

\section{Conclusions}

This paper proposes a new corner detection method based on steerable filters and Harris algorithm. As we know, steerable filters with better orientation selectivity and use of directional and band-limited filters enables us to detect true and stable corners. This new corner detection is based on multi direction levels. The proposed method detects stable and localized true corners with minimum number of false and missed corners, even after rotation, scaling, and testing number of images. 
Signal \& Image Processing : An International Journal (SIPIJ) Vol.3, No.5, October 2012

\section{REFERENCES}

[1] C. Harris and M. Stephens, "A combined corner and edge detector," in Fourth Alvey Vision Conf., pp. 147-151(1988)

[2] S. Smith and J. Brady, "SUSAN-a new approach to low-level image processing, "International. Journal of Computer Vision". 23_1_, 45-48(1997)

[3] L. Kitchen, A. Rosenfeld, Gray level corner detection, Pattern Recognition. Lett. 95-102(1982)

[4] Freeman, W.T., Adelson, E.H.: The design and use of steerable filters. IEEE Trans. Pattern Anal.Mach. Intell. 13, 891-906 (1991)

[5] Simoncelli, E.P., Freeman,W.T. "The steerable pyramid: a flexible architecture for multiscalederivative computation”In Second Int'l Conf on Image Proc, vol. 3, pp. 444-447, Washington, DC,October 1995

[6] Simoncelli, E.P., Freeman, W.T., Adelson, E.H., Heeger, D.J. "Shiftable multiscale transform". IEEE Trans. Inform. Theory 38(2), 587-607 (1992)

[7] Z.Zheng et al, "Analysis of gray level corner detection" Pattern Recognition Letters 20 (1999) 149162

[8] F. Mokhtarian, F. Mohanna "Performance evaluation of corner detectors using consistency and accuracy measures" Computer Vision and Image Understanding 102 81-94(2006)

[9] Karasaridis, A., Simoncelli, E.: A filter design technique for steerable pyramid image transforms. Proc. IEEE ICASSP 4, 2387-2390 (1996)

\section{Authors}

Mahesh received the B.E. degree from Gulbarga University, Gulbarga, Karnataka, and M.Tech degree from JNTU Hyderbad A.P. He is pursuing PhD in the area of Digital Image Processing at JNTU, Anantapur. He is working as an Associate Prof, in the Department of Electronics and Communications Engineering, Madanapalle Institute of Technology, Madanapalle. He has more than 11 years of experience in teaching field.

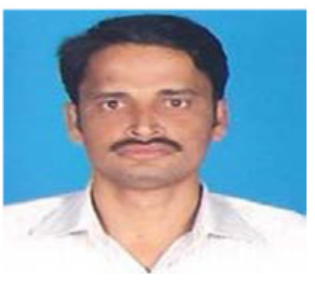

Dr.M.V. Subramanyam is the Prof of ECE \& Principal, Santhiram Engineering College, Nandyal, Kurnool Dist, A.P. He did his PhD from JNTU Hyderbad. He has more than 21 years of teaching experience with various designations in engineering colleges. He published more than 36 papers in various National and International journals and conferences. $\mathrm{He}$ is editorial member for different national and international journals. His research areas are Digital Image processing, Signal Processing and Adhoc wireless networks.

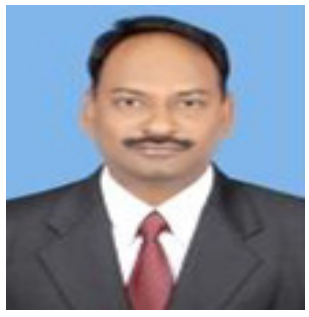

